\title{
Forgiveness and psychosocial reactions to disability: a pilot study to examine change in persons with spinal cord injury
}

\begin{abstract}
Spinal cord injuries (SCI) are among the most traumatic onset of disabilities to date. Due to the nature of spinal cord injury and how it affects the person's life and psychosocial adjustment, there are a multitude of feelings, changes, persons, situations, and transgressions that need to be resolved and forgiven. In an effort to help persons with SCI do that, two interventions - Enright's Forgiveness is a Choice intervention and Kennedy and Duff's (2001) Coping Effectively with Spinal Cord Injury training - were facilitated on-line as part of a self-study treatment format among persons with spinal cord injury. The interventions were examined to determine their ability to aid in the increase of forgiveness and changes in psychosocial reactions to disability. Findings from this study found that both interventions are applicable and helpful in assisting persons with SCI in forgiving and experiencing changes in psychosocial reactions to disability, but differences were found in long-term change.
\end{abstract}

Keywords: forgiveness, psychosocial, adjustment, disability, spinal cord injury, psychosocial reactions, interventions, coping effectively
Volume 4 Issue 4 - 2019

\author{
Susan Stuntzner,' Ruth Lynch, ${ }^{2}$ Robert Enright, ${ }^{3}$ \\ Michael Hartley, ${ }^{4}$ Angela MacDonald ${ }^{5}$ \\ 'Rehabilitation Services and Counseling, Educational Support \\ Services, University of Texas Rio Grande Valley, Southwestern \\ Oregon Community College, USA \\ ${ }^{2}$ Rehabilitation Counseling \& Special Education, Emeritus Faculty, \\ University of Wisconsin, USA \\ ${ }^{3}$ Educational Psychology, University of Wisconsin, USA \\ ${ }^{4}$ Disability \& Psychoeducational Studies, University of Arizona, USA \\ ${ }^{5}$ Rehabilitation Counseling \& Human Services, University of Idaho, \\ USA
}

Correspondence: Susan Stuntzner, Rehabilitation Services and Counseling, Educational Support Services, University of Texas Rio Grande Valley, Southwestern Oregon Community College, Tel 54I888-I578, Fax54|-888-723I,

Email Susan.stuntner@utrgv; susan.stuntner@socc.edu

Received: July 24, 2019 | Published: August 05, 2019

\section{Introduction}

Spinal cord injury (SCI) is a disability that is immediate, drastic, and forever changes a person's life and that of his or her loved ones. ${ }^{1}$ Oftentimes, SCIs occur following the event of a major traumatic event such as motor vehicle accidents, falls, acts of violence (i.e., gunshot wounds) and recreational activities (i.e., swimming accidents). ${ }^{2}$

People who acquire a SCI find themselves faced with multiple personal and life changes. People must navigate the physical and functional changes because of the injury and learn how to effectively cope with these changes (i.e., decreased mobility and sensation, alterations in bowel and bladder functioning, changes in personal independence; Falvo, 2009). ${ }^{3}$ Along with understanding the physical and functional changes, there is also a plethora of emotional and psychological reactions (i.e., grief, loss, blame, guilt, selfcondemnation, shame, helplessness, anger, depression, anxiety). ${ }^{4,5}$ A person with SCI also has to deal with societal attitudes, biases, discrimination, and social stigma; disparities in healthcare; and social isolation from peers who may also have a disability. ${ }^{6,7}$ Other changes that need to be addressed are the familial role, social support, financial, and employment issues (i.e., unemployment, employer discrimination, reduced earnings, care giving, and new or existing relationships)..$^{8-10}$

People who are not able to adjust and work around these hurts and injustices may find themselves experiencing negative emotions such as anger, resentment, depression, anxiety, loss of self-esteem, hopelessness, and frustration. . $, 4,7,11^{-}$all of which have the potential to negatively influence their psychosocial reactions to disability (Burns, Boyd, Hill, \& Hough, 2010). Individuals who are not adjusting well to the SCI may find themselves turning to drugs or alcohol in an effort to alleviate their negative thoughts and feelings. Misuse of substances is prevalent among some people with spinal cord injuries and has the ability to complicate and delay the psychological adjustment to disability process. ${ }^{13}$

\section{Relevant literature}

A number of factors or skills that aid with positive coping following disability have been discussed throughout the literature. Some factors are associated with actions and external changes such as increased levels of education or employment while others are related to emotional coping and psychological adjustment. Factors known to contribute to positive coping and psychological adjustment to disability include having social support, strong family relationships, adequate problem-solving skills, ${ }^{6,7,14}$ positive learnt behavior from past experiences, ${ }^{9}$ spiritual practices, ${ }^{15}$ meaning-making and purpose following disability, ${ }^{1}$ hope ${ }^{16}$ reduced levels of negative emotions (i.e., anger, depression, anxiety), and forgiveness. ${ }^{17,18}$ Of these factors, problem-solving skills, positive learnt behavior, spiritual practices, meaning-making and finding purpose following disability, reduced levels of negative emotions, and forgiveness are of particular interest due to the study's use of Enright's ${ }^{19}$ forgiveness intervention (i.e., Forgiveness is a Choice, FC) and Kennedy and Duff's (2001) Coping Effectively with Spinal Cord Injury Training (CET). Both the FC and the CET teach people new skills, problem-solving behaviors, how to make sense of life events, and help reduce negative emotions.

\section{Enright's forgiveness intervention (FC)}

Enright ${ }^{19}$ and Enright and colleagues ${ }^{20}$ developed a four phase, 20unit Forgiveness Intervention (FC) that has been empirically studied, 
discussed throughout the literature, and utilized as a component of the present study among persons with SCI. The model conceptualizes forgiveness as a process people go through and presents forgiveness as an approach that can be taught to help people live and heal regardless of a person's current level of functioning. The model consists of four phases: (a) Uncovering Phase, (b) Decision Phase, (c) Work Phase, and (d) Outcome Phase and allows people to work on forgiveness in an individualistic manner; meaning the process of learning to forgive needs to fit the person's specific needs and situation.

The Uncovering Phase is comprised of eight units that help people recognize emotional and psychological pain, unjust hurts and offenses, inner feelings held towards the identified offender, ways their life has been altered because of the offense, and the magnitude of injustice that has occurred. The Decision Phase consists of three units that assist people in understanding forgiveness as a viable alternative for coping with the identified hurt and offense. The Work Phase is constructed of four units that help people forgive, reframe an offending person or party, develop more compassionate and empathetic feelings towards the person and the hurtful event, and work on not retaliating or reacting negatively toward the source of inflicted pain. The Outcome Phase consists of five units where people strive to achieve a deeper understanding of forgiveness, find meaning and purpose in their hurt and pain, use the experience to help others, recognize that all people experience hurts and offenses as a part of life, and discover the positive effects of emotional and psychological healing.

Enright's Forgiveness Intervention (FC) has been studied empirically among a number of different populations, including adult incest survivors, ${ }^{21}$ partners of people who chose to have an abortion, ${ }^{22}$ persons with substance abuse issues, ${ }^{23}$ adult children of alcoholics, ${ }^{24}$ emotionally abused women, ${ }^{25}$ persons with fibromyalsia who experienced abuse, ${ }^{26}$ persons with spinal cord injury, ${ }^{27}$ and persons with coronary artery disease.$^{28}$ Overall, these empirical studies provide compelling evidence that forgiveness reduces the presence of negative thoughts and feelings following injury, illness, and disability, and also increases the presence of positive attributes (i.e., hope, self-esteem, problem-solving skills, improved personal relationships, forgiveness). In fact, a qualitative study by Willmering ${ }^{17}$ found that persons with spinal cord injury (SCI) identified forgiveness as a critical factor that aided in their adjustment and acceptance to living with SCI. Taken together, Enright's ${ }^{19}$ Forgiveness Intervention (FC) is a promising intervention to improve adaptation and coping among individuals with disabilities; however, there is a need for more research comparing it to more established interventions designed for individuals with SCI.

\section{Kennedy and Duff's coping effectively with spinal cord injury training (CET)}

Kennedy and Duff (2001) developed the Coping Effectively with Spinal Cord Injury training (CET) as a seven-module intervention to support people with spinal cord injury (SCI). Specifically, the CET intervention is designed to teach people with SCI about stress and stressors and the relationship between how they appraise them and the stress experienced, including the importance of specific versus global stressors and identification of those that can be modified, coping skills and problem-solving strategies, reduction of negative coping strategies (i.e., cognitive, emotional, behavioral), differentiation between adaptive and maladaptive coping skills, and development and maintenance of social support. Throughout the course of the CET intervention, people are provided with opportunities to identify and individualize the information and exercises to their specific situation so they can personally determine what causes them stress, how they react to stress, and what they can do to change their predicament and improve coping.

Kennedy and Duff's (2001) Coping Effectively with Spinal Cord Injury Training (CET) has been empirically studied among persons with spinal cord injuries. ${ }^{5,27,29,30,31}$ Results from these studies show that CET is effective in reducing anxiety and depression ${ }^{30,31}$ and promoting change in self-perception between individuals' 'ideal' self and 'real' self. ${ }^{30}$ Similarly, Stuntzner et al. ${ }^{27}$ found that CET was effective in reducing depression and state anxiety from pre-test to post-test and in reducing depression, anxiety, and trait anger from pre-test to followup. Furthermore, Duchnick et al. ${ }^{29}$ found that CET decreased anxiety and depression and promoted higher levels of adjustment to disability among persons with SCI when compared to people who were a part of a therapy support group. Taken as a whole, CET is an effective intervention to improve coping and adaptation following SCI.

\section{Purpose of Study}

The purpose of the study was to examine the comparability of Enright's ${ }^{19}$ Forgiveness is a Choice (FC) intervention to Kennedy and Duff's (2001) Coping Effectively Training (CET) among persons with spinal cord injury (SCI) in the increase of forgiveness and changes in psychosocial reactions to disability. Furthermore, while online interventions have become more common over the past decade; ${ }^{32-34}$ researchers have yet to compare the viability of the delivery of either intervention to individuals with SCI via an online format rather than in-person. Online delivery is important because some people with SCI may live in rural locations and may have mobility or travel issues, may not have adequate exposure to services, or may lack access to services that are affordable and lower cost. ${ }^{35}$ Since little is known about how the online delivery of these two interventions compare to one another in the promotion of forgiveness or changes in psychosocial reactions to disability, the research questions examined were: (1) will there be statistically significant differences in measures of forgiveness and psychosocial reactions to disability from pre-test to post-test for both interventions; (2) will the pattern of statistically significant differences be comparable between the two interventions at pre-test and posttest as well as pre-test to follow-up? The hypothesis was that the Forgiveness Intervention (FC) would have comparable change scores to the Coping Effectively with Spinal Cord Injury Training (CET).

\section{Methods}

Replicating the procedures used in previous intervention studies on forgiveness. ${ }^{21,22,26,25}$ participants were recruited and then randomly assigned to either the FC group or the CET group. All of the participants completed an 8-week, online intervention based on either Enright's ${ }^{19}$ Forgiveness is a Choice: A Step-by-Step for Resolving anger and Restoring Hope or Kennedy and Duff's (2001) Coping Effectively with Spinal Cord Injury. Having access to the designated books and manuals was essential as this study was delivered as an online, selfstudy treatment model rather than an online group format. Participants were assigned a code and password to enable them to gain access to the study website, securely communicate with the researcher and submit weekly assignments, and make sure participants did not have access to other participants' information. All participants completed the Enright Forgiveness Inventory ${ }^{36}$ and Reaction to Illness and Disability Inventory ${ }^{37}$ pre-test, post-test, and at a two-month followup in order to compare effectiveness of the two interventions. 


\section{Participants}

Participants were recruited nationally from disability agencies and disability-related websites including: Model Spinal Cord Injury Centers, centers for independent living, vocational rehabilitation division, Paralyzed Veterans of America, hospitals, rehabilitation agencies, NSCIA Chapter Network, local university disability centers, spinal cord injury support groups, and spinal cord injury on-line list serves. Prior to being accepted into the study, potential participants were asked to fill out a demographic questionnaire and a participant psychological screening form. To be eligible, participants had to have a spinal cord injury for at least one year, be at least 18-years of age, and not report a current problem with alcohol or substance use. Screening for alcohol and substance use was considered important due to the reported prevalence of alcohol or substance use among persons with spinal cord injury. To be successful in this study, the researcher felt it was important to ensure that people did not have current substance abuse issues prior to being admitted into the study.

Sixteen participants were selected and randomly assigned to the FC group $(\mathrm{N}=9)$ or CET group $(\mathrm{N}=7)$. All of the participants completed the pre- and post-test, but only 11 completed the follow-up $(\mathrm{N}=6$, forgiveness group; $\mathrm{N}=5 \mathrm{CET}$ ). Participants who did not complete the follow-up did not provide a reason, but it was observed by the researcher that some participants reported the nature of working on forgiveness to be challenging. Therefore, it is possible that some felt they had done enough personal work following the conclusion of the interventions. Collectively, participants ranged in age from 37 to 54 years $(M=46.0$ years of age, $\mathrm{SD}=5.1)$. Both groups had participants who were of similar ages (37-54 years, FC; $38-54$ years, CET). Time since injury ranged from 1.5 to 29 years and significant differences between groups were not found $(M=17.0$ years, $S D=11.8, \mathrm{FC} ; M$ $=10.0, S D=8.1$, CET).

\section{Instrumentation}

\section{Enright Forgiveness Inventory}

The Enright Forgiveness Inventory (EFI) is a 60-item selfreport inventory used to measure a person's level of interpersonal forgiveness. ${ }^{22}$ The EFI requires 40 minutes to complete and a minimum of a $5^{\text {th }}$ grade reading level. When calculating an overall total forgiveness score, low scores (i.e.,60) represent a low level of forgiveness and high scores (i.e., 360) indicate high levels of forgiveness. ${ }^{36}$ The EFI has strong support for both reliability and validity. Subkoviak et al. ${ }^{37}$ report internal consistency coefficients to range from 0.93 to 0.98 . Enright et al. ${ }^{36}$ refer to the EFI's testretest reliability as ranging from 0.67 to 0.91 . Furthermore, subscale correlations have been reported to range between 0.80 and $0.87 .{ }^{38}$ An examination of the pre-test scores in the present study found an internal consistency and reliability Chronbach Alpha value of 0.98 across the 60 -items.

\section{Reaction to Illness and Disability Inventory}

The Reaction to Illness and Disability Inventory (RIDI) is a 60item instrument constructed to assess reactions to physical disability. ${ }^{37}$ The RIDI contains eight psychosocial reactions: Shock, Anxiety, Denial, Depression, Internalized Anger, Externalized Hostility, Acknowledgement, and Adjustment. ${ }^{39}$ Each scale contains items which can be rated from " 1 " to " 4 " with 1 being equivalent to "Never" and 4 being the same as "Often". ${ }^{37,39}$ Previous research indicated
Chronbach alphas of the eight psychosocial reactions (Shock= 0.79 , Anxiety $=0.83$, Denial $=0.64$, Depression $=0.88$, Internalized Anger $=0.81$, Externalized Anger $=0.84$. Acknowledgment $=0.78$, and Adjustment $=0.89 .{ }^{38}$ In the present study, an examination of the pretest scores for internal consistency found the following Chronbach Alphas: Shock $=0.68$, Anxiety $=0.84$, Denial $=0.48$, Depression $=0.74$, Internalized Anger $=0.81$, Externalized Hostility $=0.73$, Acknowledgement $=0.61$, Adjustment $=0.77$.

\section{Analysis}

Data was analyzed by using sample t-tests to determine change in the mean scores of each intervention group. Means were calculated from pre-test to post-test as well as pre-test to follow-up, allowing for a focused comparison of how participants' scores on the forgiveness and psychosocial reactions to disability changed between the two intervention groups. Questions one and two used two-tailed independent sample $\mathrm{t}$-tests to examine if changes in forgiveness and psychosocial reactions to disability were comparable between the two intervention groups from pre-test to post-test. Question three used group mean scores and analyzed data according to a paired sample t-test to explore long-term changes at follow-up for either intervention group.

\section{Results}

The first research question focused on within group differences in the Forgiveness Intervention (FC) group and the Coping Effectively with Spinal Cord Injury Training (CET) group on the Enright Forgiveness Inventory ${ }^{36}$ and Reaction to Illness and Disability Inventory ${ }^{37}$ from pre-test to post-test. The groups were therefore first examined separately to determine effectiveness in promoting change in forgiveness and psychosocial reactions to disability scores from pretest to post-test. Results indicated that the FC group improved their mean EFI forgiveness score by 82.1 points, from 148.33 to 230.44 points, and achieved statistical significance $t(8)=-5.086, p<.05$, while the CET group increased by 40.0 points, from 176.57 to 216.57 points, and also achieved statistical significance $t(6)=-3.568, p<.05$. As for the eight Reaction to Illness and Disability Inventory (RIDI) subscales, the FC group reduced their post-test scores enough to achieve statistical significance in Shock $t(8)=3.192, p<.05$, Anxiety $t(8)=2.242, p<.05$, Denial $t(8)=2.268, p<.05$, Depression $t(8)=2.335$, $p<.05$, Internalized Hostility $t(8)=2.910, p<.05$, and Externalized Anger $t(8)=2.578, p<.05$, while the CET group affected post-test scores enough to only achieve statistical significance in Adjustment $t(6)=-3.057, p<.05$ (Table 1) (Table 2). Results of the present study therefore found that while both groups increased in the EFI forgiveness measure, the FC group had statistically significant decreases in the negative emotional reactions of the RIDI subscales: Shock, Anxiety, Denial, Depression, Internalized Hostility, and Externalized Anger, while the CET group only had a statistical change in Adjustment. Interestingly, the FC group did not achieve statistical change in either Acknowledgement or Adjustment despite the significant decrease in virtually all of the negative psychosocial reactions to disability.

The second research question compared the effectiveness of the FC and CET interventions. The hypothesis was that the interventions would be comparable, and the results found this to be true. Although the FC group had a 42 point greater increase in mean scores compared to the CET group on the EFI forgiveness measure, the difference was not statistically significant and the FC and CET interventions were found to be comparable in increasing forgiveness (Table 3). 
In addition, the FG group demonstrated larger amounts of change from pre-test to post-test than the CET group on some psychosocial reactions to disability and not on others; yet, when change scores were compared between the interventions, no significant differences were found. Change and comparison scores are reflected in Table 3 and included the following: Shock, a 1.73 point reduction, $t(14)=1.405$, $p>.05$, Anxiety, a 1.05 point decrease, $t(14)=.565, p>.05$, Denial, a 1.14 point difference, $t(14)=1.580, p>.05$, Depression, a 0.98 point decrease, $t(14)=.622, p>.05$. Internalized Anger, a 4 point decrease, $t(14)=1.835, p>.05$, and Externalized hostility, a 3.25 point difference, $t(14)=1.948, p>.05$. Acknowledgment and Adjustment change scores were slightly higher in the CET group. Scores on Acknowledgement reflect a 0.68 point increase, $t(14)=.361, p>.05$ and Adjustment, a 0.57 point increase, $t(14)=.388, p>.05$ compared to the FC group. Overall, comparison of change scores from pre-test to post-test did not demonstrate a statistical significance between the two intervention groups, thus indicating that the $\mathrm{FC}$ intervention is comparable to the CET intervention in influencing alterations in psychosocial reactions to disability as well as forgiveness (Table 3 ).

Table I Forgiveness Group (FC) scores at pre-test and post-test

\begin{tabular}{|c|c|c|c|c|}
\hline & \multicolumn{2}{|c|}{ Pretest $(n=9)$} & \multicolumn{2}{|c|}{ Posttest $(n=9)$} \\
\hline & Mean & SD & Mean & SD \\
\hline & 148.33 & 51.93 & $230.44 *$ & 64.63 \\
\hline Shock & 15.00 & 4.03 & $12.55^{*}$ & 3.16 \\
\hline Anxiety & 15.55 & 3.94 & $13.22^{*}$ & 3.80 \\
\hline Denial & 10.22 & 2.16 & $9.22 *$ & 1.48 \\
\hline Depression & 17.66 & 3.35 & $|5.1|^{*}$ & 3.01 \\
\hline Internalized Anger & 20.11 & 2.89 & $16.11^{*}$ & 3.82 \\
\hline Externalized Hostility & 16.55 & 4.24 & $13.44^{*}$ & 3.94 \\
\hline Acknowledgement & 23.22 & 3.59 & 23.11 & 2.36 \\
\hline Adjustment & 24.55 & 0.88 & 26.55 & 3.20 \\
\hline
\end{tabular}

Note $*_{p}<.05$

Table 2 Coping effectively with spinal cord injury (CET) scores at pre-test and post-test

\begin{tabular}{lllll}
\hline & \multicolumn{2}{c}{ Pre-test $(\mathbf{n}=7)$} & \multicolumn{2}{l}{ Post-test $(\mathbf{n}=\mathbf{7})$} \\
\cline { 2 - 5 } & Mean & SD & Mean & SD \\
\hline Forgiveness & 176.57 & 56.98 & $216.57^{*}$ & 65.44 \\
$\begin{array}{l}\text { Psychosocial Reactions } \\
\text { Shock }\end{array}$ & 12.71 & 2.87 & 12.00 & 3.16 \\
Anxiety & 14.57 & 2.50 & 13.28 & 3.16 \\
Denial & 8.85 & 1.06 & 9.00 & 1.63 \\
Depression & 17.85 & 2.79 & 16.28 & 1.70 \\
Internalized Anger & 14.71 & 4.88 & 14.71 & 5.76 \\
$\quad$ Externalized & 12.28 & 3.14 & 12.42 & 2.22 \\
Hostility & 23.00 & 3.69 & 23.55 & 2.07 \\
Acknowledgement & 23.00 & 5.25 & $25.57^{*}$ & 2.07 \\
Adjustment & 25
\end{tabular}

Note $* p<.05$
Table 3 Comparison in change scores between forgiveness group (FC) and coping effectively with spinal cord injury group (CET) from Pre-Test to Posttest

\begin{tabular}{llllll}
\hline & \multicolumn{2}{l}{$\begin{array}{l}\text { Forgiveness } \\
\text { group }\end{array}$} & $\begin{array}{l}\text { Coping } \\
\text { effectively } \\
\text { group }\end{array}$ & $\begin{array}{l}\text { Comparison } \\
\text { in change } \\
\text { scores }\end{array}$ \\
\cline { 2 - 6 } & Mean & SD & Mean & SD & t \\
\hline $\begin{array}{l}\text { Forgiveness } \\
\begin{array}{l}\text { Psychosocial } \\
\text { Reactions }\end{array}\end{array}$ & 82.11 & 48.42 & 40.00 & 29.66 & 2.016 \\
Shock & -2.44 & 2.29 & -0.71 & 2.62 & 1.405 \\
Anxiety & -2.33 & 3.12 & -1.28 & 4.30 & .565 \\
Denial & -1.00 & 1.32 & 0.14 & 1.57 & 1.580 \\
Depression & -2.55 & 3.28 & -1.57 & 2.93 & 0.622 \\
Internalized Anger & -4.00 & 4.12 & 0.00 & 4.58 & 1.835 \\
Externalized & -3.11 & 3.62 & 0.14 & 2.85 & 1.948 \\
Hostility & -.011 & 3.17 & 0.57 & 4.39 & 0.361 \\
Acknowledgement & -1.35 & 2.57 & 2.22 & 0.388 \\
Adjustment & 2.00 & 3.35 & & & \\
\hline
\end{tabular}

Note $*_{p}<.05$

Research question three assessed if participants from either group demonstrated long-term change in forgiveness and psychosocial reactions to disability pre-test to follow-up. Analysis required that means, change scores from pre-test to follow-up, and standard deviations be determined for the dependent variables of forgiveness and psychosocial reactions to disability. Scores from both interventions were examined separately to determine whether either one had longterm change effects, eight-weeks following the completion of the posttests. Since this type of inquiry had not previously been conducted, it was not known if one intervention would perform better long-term than the other, thus, it was hypothesized that either intervention would indicate long-term change in forgiveness and psychosocial reactions to disability.

Results of this study found that both interventions achieved differences in long-term change at follow-up. Participants in the FC group maintained and increased their forgiveness score to 233.33 points at follow-up by 85 points, thus demonstrating a statistically significant level of change, $t(5)=-3.895, p<.05$. Participants in the coping intervention group had a follow up score of 201.60 which was a change of 25.03 points from the start of the study. However, the change was a slight decrease from the scores achieved at post-test and were not clinically significant, $t(4)=-1.233, p>.05$.

In regards to psychosocial reactions, long-term change from pre-test to follow-up showed varied results for both intervention groups. Holistically, both intervention groups showed statistically significant, long-term change in four psychosocial reactions. The FC group demonstrated statistically significant change in Shock, a 4.17 point reduction, $\mathrm{t}(5)=2.166, \mathrm{p}<.05$, Denial, a 0.89 point decrease, $\mathrm{t}(5)=2.150, \mathrm{p}<.05$, Internalized Anger, a 5 point decrease, $\mathrm{t}(5)=$ $2.221, \mathrm{p}<.05$, and Externalized Hostility, a 4.72 point reduction, $\mathrm{t}$ $(5)=2.782, \mathrm{p}<.05$; while, those from the CET group showed statistically significant change in Shock, a 1.71 point decrease, $t(4)=7.483, p<.05$, Depression, a 5.45 point decrease, $\mathrm{t}(4)=6.410, \mathrm{p}<.05$, Externalized Hostility, a 3.28 point reduction, $\mathrm{t}(4)=2.256, \mathrm{p}<.05$, and Adjustment, a 2 point increase, $\mathrm{t}(4)=-7.060, \mathrm{p}<.05$. Also of interest is the fact that although participants from the forgiveness group did not reach 
statistically significance in Anxiety, Depression, Acknowledgement or Adjustment, they did maintain or improve change in their scores at follow-up.

\section{Discussion}

Overall, the results of the present study add evidence that forgiveness is important to the positive psychological adjustment following the onset of spinal cord injury (SCI). The first question wanted to know if either of the two interventions affected change in forgiveness from pre-test to post-test. Data derived from the study indicated that the first question was successfully met and achieved the desired results. Results from the FC group indicated that participants improved their forgiveness scores from pre-test to post-test by 82.11 points. This increase in forgiveness was found to be statistically significant. The CET group increased its forgiveness scores by 40 points and was found to be statistically significant. Although the FC group had a larger increase in forgiveness from pre-test to post-test, changes in forgiveness scores were considered comparable to the CET group. Previous forgiveness studies among persons with spinal cord injury have found forgiveness to be beneficial to increase their quality of life ${ }^{18}$ and to possibly aid in the adjustment to disability process, ${ }^{17}$ but this is the first study to be conducted among this population utilizing Enright's ${ }^{19}$ FC model. Similarly, this is the first study to examine Kennedy and Duff's (2001) CET intervention in relation to changes in forgiveness. Since this is the first study to examine change in forgiveness utilizing these two interventions and due to the small sample size, additional research is warranted and needed to further substantiate the findings.

In addition to forgiveness, the present study examined whether the $\mathrm{FC}$ group or the CET group would effect change in psychosocial reactions to disability from pre-test to post-test. Findings from both groups provide partial support for this question. Of particular interest is not only the psychosocial changes that occurred within each group, but the fact that each intervention led to different changes in psychosocial reactions to disability. More specifically, the FC group achieved statistical significance in reducing the negative psychosocial reactions to disability, which included reductions in Shock, Anxiety, Denial, Internalized Anger, and Externalized Hostility, however, did not reach statistical significance in alterations pertaining to Acknowledgment, and Adjustment.

Considerations of these reactions are either new or related to other research conducted among persons with spinal cord injury. To date, this is the first research study to examine and find a significant relationship between Enright's Forgiveness Intervention and a reduction in Shock, Denial, Internalized Anger, or Externalized Hostility among individuals with disabilities. The present study adds evidence that forgiveness can aid in the reduction of negative emotions among persons with spinal cord injury, ${ }^{27}$ but further research is suggested to strengthen and substantiate the findings.

Another important finding is that the CET group did not demonstrate statistical significance in reducing Shock, Anxiety, Denial, Depression, Internalized Anger, Externalized Hostility, or Acknowledgement, but they did substantially increase on Adjustment. Findings from this group are somewhat surprising given the previous research studies that utilized CET among persons with spinal cord injury and found it to aid the reduction of anxiety and depression. $5,30,31$ To date, little is known about the effect of CET on adjustment to disability or psychosocial reactions to disability among persons with disabilities. The studies conducted are by Duchnick et al. ${ }^{29}$ These scholars found that CET and an alternative treatment group did not significantly differ in regards to adjustment to disability at 3-month follow-up post-discharge. Besides the current study, research has not been conducted to determine changes psychosocial reactions to disability through the use of CET; thus, there are not any studies to compare these findings too.

Also of interest are the findings of these two intervention groups compared. Although both groups reported some change in different psychosocial reactions to disability, and despite the fact that statistical significance was not found in all eight areas, results suggest that both the FC group and the CET group were comparable in the changes reported; thus, suggesting that there were not any significant changes found between the groups.

Question three examined differences in long-term changes from pre-test to follow-up in forgiveness and psychosocial reactions to disability. Thus, this question was explored to determine if the FG group or the CET group effected long-term change, 2 months following completion of the designated intervention. Both intervention groups demonstrated partial support for the maintenance and increase in forgiveness at follow-up. The FC group increased its gain score to 85 points and the CET increased its gain score by 25.03 . Gains made by the FG group are slightly higher than reported at post-test and were found to be statistically significant. However, gains made by the CET group decreased from post-test and were not found to be statistically significant. Despite this decrease, forgiveness scores were slightly higher than at the start of the study. Similar to question one, little is known about the effects of the FC intervention or the CET intervention in effecting long-term change in forgiveness among persons with spinal cord injury; thus, additional research is suggested to further substantiate these findings and to learn about the effect of these interventions on the promotion of forgiveness.

Differences and variations in changes pertaining to psychosocial reactions to disability were found between the two intervention groups. More specifically, the FC group demonstrated significant changes in Shock, Denial, Internalized Anger, and Externalized Hostility. These findings indicate that prior changes reported at posttest were maintained in these areas. Significant changes were not found in Anxiety, Depression, Acknowledgement, or Adjustment; however, change scores from pre-test to follow-up indicate reductions in Anxiety and Depression and increases in Adjustment, which might support the possibility of a trend of change occurring. Participants in the CET group were found to have significant changes in Shock, Depression, Externalized Hostility, and Adjustment, but not in Anxiety, Denial, Internalized Anger, and Acknowledgement. Findings from the CET demonstrate more activity and change in some of the negative emotional reactions from those first acquired at post-test. Reasons for these changes are not known but may be related to the possibility that over the 2-month break, participants had time to absorb, practice, and integrate the information and skills learned while completing the intervention phase of the study. Changes in Anxiety and Denial while not statistically significant were found to be more prevalent, based on mean change scores, at follow-up than reported at post-test; thus possibly suggesting a trend of change in some of the negative psychosocial reactions to disability.

\section{Limitations}

A number of limitations exist which may be considered as additional research is warranted. Similar to previous forgiveness intervention research, the first limitation is sample size and lack of 
cross-validation studies to compare the study to as it relates to persons with disabilities..$^{21,22,25,26}$ To be sure, the results of the present study would be strengthened through further research among persons with spinal cord injury and the acquisition of larger sample sizes. Having a larger number of participants could be helpful in determining generalizability of results and comparison of interventions to this population. For these reasons, the findings from this study should be considered preliminary and as a starting place for further exploration of these interventions among persons with spinal cord injury.

The second limitation is the wide range of how long participants had a spinal cord injury, ranging from 1.5 to 29 years of age. While both intervention groups were relatively similar as far as mean and range of time since injury, it would be useful to examine if forgiveness is an intervention that is more appropriate earlier or later in adjustment process and were not found to be significantly different. Further study is needed to understand how time since injury correlates with forgiveness. The third limitation relates to the length of the study and the lack of knowledge known about whether or not the changes reported at follow-up were maintained long-term past the two month period of time. To date, intervention studies typically consist of a pretest, post-test or a pre-test, post-test, follow-up design. Studies that have a follow-up phase often occur 8 to 12 weeks after the completion of the post-test, but few studies exist that contain long-term data. Future research is warranted to help determine if changes that occur at post-test or follow-up are maintained a year later.

The final limitation is the possibility of selection bias among participants. As previously indicated, the aforementioned study was conducted on-line rather than face-to-face. Research is beginning to emerge that support the viability and comparability of face-to-face versus online treatment studies (see Schoenberg et al., 2008), yet, for purposes of this study it is possible that some participants were specifically interested in it because it was on-line rather than face-toface. For this reason, results may be the bi-product of selection bias that occurred among the participants interested in it.

\section{Conclusion}

Interventions to increase a person's coping abilities are needed to help individuals with SCI improve mental and emotional functioning. Two interventions - Enright's ${ }^{19}$ Forgiveness is a Choice intervention and Kennedy and Duff's (2001) Coping Effectively with Spinal Cord Injury training - were administered and explored among persons with SCI to help determine their effectiveness in creating positive changes in forgiveness and psychosocial reactions to disability. Results from this study show partial support for both interventions in increasing forgiveness and effecting change in some of the phases of psychosocial reactions to disability. Because few studies have been done to examine the effect of forgiveness or compare these two interventions as strategies to increase peoples' forgiveness and/or psychosocial reactions to disability, further research is warranted to substantiate and expand these findings. ${ }^{40}$

\section{Funding}

None

\section{Acknowledgements}

None.

\section{Conflicts of interest}

The author declares no conflict of interest.

\section{References}

1. Roon-Cassini T, de St Aubin E, Valvano A, et al. Psychological wellbeing after spinal cord injury. Rehabilitation Psychology. 2009; 53(4):306-314.

2. National Spinal Cord Injury Statistical Center. National spinal cord injury (SCI) facts and figures; 2016.

3. Falvo DR. Conditions of the nervous system: Part II. Spinal cord, peripheral nervous system, and neuromuscular conditions. Medical and psychosocial aspects of chronic. 2009.

4. Cao Y, DiPiro ND, Xi J, et al. Unmet expectations of adjustment and depressive symptoms among people with chronic traumatic spinal cord injury. Rehabilitation Psychology. 2014;59(3):313-320.

5. Kennedy P, Duff J, Evans M, et al. Coping effectiveness training reduces depression and anxiety following traumatic spinal cord injuries. British Journal of Clinical Psychology. 2003;42:41-52.

6. Bogart KR. The role of disability self-concept in adaptation to congenital or acquired disability. Rehabilitation Psychology. 2014; 59(1):107-115.

7. Martz E, Livneh H. Coping with spinal cord injuries. In: E Martz, H Livneh, editors. Coping with chronic illness and disability. New York, NY: Springer. 2007;363-387.

8. Chan RC, Lee PW, Lieh-Mak F. The pattern of coping in persons with spinal cord injuries. Disability and Rehabilitation. 2000;22(11):501 -507 .

9. Power PW, Dell Orto AE. Impact of illness and disability on adults. Families living with chronic illness and disability. NY: Springer. New York;2004;48-69.

10. Thompson NJ, Coker J, Krause JS, et al. Purpose in life as a mediator of adjustment after spinal cord injury. Rehabilitation Psychology. 2003;48(2):100-108

11. Saunders LL, Krause JS, Focht KL. A longitudinal study of depression in survivors of spinal cord injury. Spinal Cord. 2012;50(1):72-7.

12. Burns SM, Boyd BL, Hill J. Psychosocial predictors of employment status among men living with spinal cord injury. Rehabilitation Psychology. 2010;55(1):81-90.

13. Heinemann AW. Spinal cord injury. In: AJ Goreczny, editor. Handbook of health and rehabilitation psychology. New York, NY: Plenum Press. $1195 ; 341-360$.

14. Elliott TR, Godshall FJ, Herrick SM, et al. Problem-solving appraisal and psychological adjustment following spinal cord injury. Cognitive Therapy and Research. 1991;15:387-389.

15. Johnstone B, Yoon DP. Relationships between the brief multidimensional measure of religiousness/spirituality and health outcomes for a heterogenous rehabilitation population. Rehabilitation Psychology. 2009;54(4):422-431.

16. Miller-Smedema S, Chan J, Phillips B. Core self-evaluations and Snyder's Hope Theory in persons with spinal cord injuries. Rehabilitation Psychology. 2014;59(4):399-406.

17. Willmering PP. Forgiveness as a self-reported factor in adjustment to disability [Doctoral dissertation, University of Wisconsin - Madison]. Dissertation Abstracts International. 1999;60(6-B):3009.

18. Webb JR1, Toussaint L, Kalpakjian CZ, et al. Forgiveness and health outcomes among persons with spinal cord injury. Disability and Rehabilitation. 2010;32(5):360-366.

19. Enright RD. Forgiveness is a choice: A step-by-step process for resolving anger and restoring hope. Washington, DC: American Psychological Association. 2001 
20. Enright RD, Freedman S, Rique J. The psychology of interpersonal forgiveness. In RD Enright \& J North, editors. Exploring forgiveness. Madison, WI: University of Wisconsin Press. 1998;46-62.

21. Freedman SR, Enright RD. Forgiveness as an intervention goal with incest survivors. Journal of Consulting and Clinical Psychology. 1996;64(5):983-992.

22. Coyle CT, Enright RD. Forgiveness intervention with post-abortion men. Journal of Consulting and Clinical Psychology. 1997;65(6): 1042-1045.

23. Lin W, Mack D, Enright RD, et al. Effects of forgiveness therapy on anger, mood, and vulnerability to substance abuse among inpatient substance-dependent clients. J Consult Clin Psychol. 2004;72(6):11141121.

24. Osterndorf CL, Enright RD, Holter AC, et al. Treating adult children of alcoholics through forgiveness therapy. Alcoholism Treatment Quarterly. 2011;29(3):274-292.

25. Reed GL, Enright RD. The effects of forgiveness therapy on depression, anxiety, and posttraumatic stress for women after spousal emotional abuse. J Consult Clin Psychol. 2006;74(5):920-929.

26. Lee YR, Enright RD. A forgiveness intervention for women with fibromyalgia who were abused in childhood. Spirituality in Clinical Practice. 2014;1(3):203-217.

27. Stuntzner S, Hartley M, Lynch R, et al. Comparison of two on-line interventions to cope with spinal cord injury: A pilot study. Annals of Psychotherapy and Integrative Health. 2015;1-20.

28. Waltman MA, Russell DC, Coyle CT, et al. The effects of a forgiveness intervention among patients with coronary artery disease. Psychology and Health. 2009;24(1):11-27.

29. Duchnick J, Letsch E, Curtiss G. Coping effectiveness training during acuterehabilitation of spinal cord injury/dysfunction. Rehabil Psychol. 2009;54(2):123-132.

30. Kennedy P, Taylor NM, Duff J. Characteristics predicting effective outcomes after coping effectiveness training for patients with spinal cord injuries. Journal of Clinical Psychology in Medical Settings. 2005;12(1):93-98.
31. King C, Kennedy P. Coping effectiveness training for persons with spinal cord injury: preliminary results of a controlled trial. $\mathrm{Br} J$ Clin Psychol. 1999;38(Pt 1):5-14.

32. Hadjistavropoulos HD, Alberts NM, Nugent M, et al. Improving access to psychological services through therapist-assisted, internet-delivered cognitive behavioral therapy. Canadian Psychology. 2014;55(4):303311.

33. Hopps SL, Pepin M, Boisvert JM. The effectiveness of cognitivebehavioral group therapy for loneliness via interrelay-chat among people with physical disabilities. Psychotherapy: Theory. 2003;40(1-2):136147.

34. Schoenberg M, Ruwe W, Dawson K, et al. Comparison of functional outcomes and treatment cost between a computer-basedcognitive rehabilitation teletherapy program and a face-to-face rehabilitation program. Professional Psychology: Research and Practice. 2008;39(2):169-175.

35. Migone P. Psychoanalysis on the Internet: A discussion of its theoretical implications for both online and offline therapeutic technique. Psychoanalytic Psychology. 2013;30(2):281-299.

36. Enright RD, Rique J, Coyle CT. The Enright forgiveness inventory user's manual. Madison, WI: The International Forgiveness Institute. 2000.

37. Subkoviak MJ, Enright RD, Wu CR, et al. Measuring interpersonal forgiveness inlate adolescence and middle adulthood. Journal of Adolescence. 1995;18(6):641-655.

38. Livneh H, Antonak RF. Reactions to disability: An empirical investigation of their nature and structure. Journal of Applied Rehabilitation Counseling. 1990;21(4):13-21.

39. Livneh H, Antonak RF. Psychosocial reactions to disability: A review and critique of the literature. Critical Reviews in Physical and Rehabilitation Medicine. 1994;6:1-100.

40. Enright RD, Coyle CT. Researching the process model of forgiveness with psychological interventions. In: EL. Worthington. Editor. Dimensions of forgiveness: Psychological research and theological perspectives London: Templeton. 1998;139-161. 